\title{
The Dual Description of Long Distance QCD and the Effective Lagrangian for Constituent Quarks
}

\author{
M. Baker \\ University of Washington, Seattle, WA 98105
}

\begin{abstract}
We describe long distance QCD by a dual theory in which the fundamental variables are dual potentials coupled to monopole fields and use this dual theory to determine the effective Lagrangian for constituent quarks. We find the color field distribution surrounding a quark anti-quark pair to first order in their velocities. Using these distributions we eliminate the dual potentials and obtain an effective interaction Lagrangian $L_{I}\left(\vec{x}_{1}, \vec{x}_{2} ; \vec{v}_{1}, \vec{v}_{2}\right)$ depending only upon the quark and anti-quark coordinates and velocities, valid to second order in their velocities. We propose $L_{I}$ as the Lagrangian describing the long distance interaction of constituent quarks.
\end{abstract}

\section{Introduction}

In 1976 Mandelstam and 't Hooft 1 proposed that the force between a quarkantiquark pair is analogous to the force between a monopole anti-monopole pair in a superconductor. A dual Meissner effect prevents the electric color flux from spreading out as the distance between the quark anti-quark pair increases. As a result a linear potential develops which confines the quarks in hadrons. A concrete model for this "dual superconductor" mechanism is realized by a dual theory 2 defined by a Lagrangian density $\mathcal{L}_{\text {eff }}\left(C_{\mu}, B_{i}\right)$ in which the fundamental variables are dual potentials $C_{\mu}$ coupled to monopole fields denoted by $B_{i}$. The local coupling of monopole fields to dual potentials gives rise to a $C_{\mu}$ mass and consequently to a dual Meissner effect. The Lagrangian density $\mathcal{L}_{\text {eff }}\left(C_{\mu}, B_{i}\right)$ of the dual theory describes the same long distance physics as the original QCD Lagrangian density $\mathcal{L}_{\mathrm{QCD}}$ defined in terms of Yang Mills potentials $A_{\mu}$ which are strongly coupled at long distances.

In this talk we construct $\mathcal{L}_{\text {eff }}$, treating quarks as classical point particles having coordinates $\vec{x}_{i}(t)$ and velocities $\vec{v}_{i}(t)$. We solve the resulting classical field equations for $C_{\mu}$ in the presence of a quark anti-quark pair to first order in their velocities. Using these solutions we eliminate the dual potentials $C_{\mu}$ from $\mathcal{L}_{\text {eff }}$ and obtain an effective interaction Lagrangian $L_{I}\left(\vec{x}_{1}, \vec{x}_{2} ; \vec{v}_{1}, \vec{v}_{2}\right) \equiv \int d \vec{x} \mathcal{L}_{\text {eff }}$ depending upon the quark and antiquark positions and velocities, valid to second order in these velocities. It contains

\footnotetext{
*Work supported in part by the U.S. Dept. of Energy under Contract No. DE-FG06-91ER40614

$\dagger$ Talk given by M. Baker at the International Workshop on Color Confinement and Hadrons, March 22-24, 1995, Osaka, Japan.
} 
two parameters $\alpha_{s}$ and the string tension $\sigma$. We use $L_{I}$ and the canonical formalism to construct the quark anti-quark Hamiltonian and obtain a specific quark model for calculating the meson spectrum. 2

We begin by showing that dual potentials give an alternate formulation of classical electrodynamics. This gives an example where the dual theory and the original Maxwell theory describe the same physics at all distances in a situation where the original Maxwell potentials and the dual potentials can have different long distance behavior.

\section{Dual Potentials In Electrodynamics}

The inhomogeneous Maxwell equations for a pair of oppositely charged particles moving in a relativistic dielectric medium are:

$$
\vec{\nabla} \cdot \vec{D}=\rho, \quad \vec{\nabla} \times \vec{H}=\vec{J}+\frac{\partial \vec{D}}{\partial t}
$$

where

$$
\begin{gathered}
\rho(\vec{x}, t)=e\left[\delta\left(\vec{x}-\vec{x}_{1}(t)\right)-\delta\left(\vec{x}-\vec{x}_{2}(t)\right)\right] \\
\vec{J}(\vec{x}, t)=e\left[\vec{v}_{1}(t) \delta\left(\vec{x}-\vec{x}_{1}(t)\right)-\vec{v}_{2}(t) \delta\left(\vec{x}-\vec{x}_{2}(t)\right)\right] .
\end{gathered}
$$

Introduce a line of polarization $\vec{P}_{s}(\vec{x}, t)$ connecting the particles (a Dirac string):

$$
\vec{P}_{s}(\vec{x}, t)=e \int_{\vec{x}_{2}(t)}^{\vec{x}_{1}(t)} d \vec{y} \delta(\vec{x}-\vec{y}(t))
$$

where the integral $d \vec{y}$ is along any path $L(t)$ connecting $\vec{x}_{1}(t)$ and $\vec{x}_{2}(t)$. (See Fig. 1.)

Fig. 1. Dirac string connecting oppositely charged particles

As the particles move, so does the Dirac string. There results a magnetization $\vec{M}_{s}(\vec{x}, t)$,

$$
\vec{M}_{s}(\vec{x}, t)=\int_{\vec{x}_{2}(t)}^{\vec{x}_{1}(t)} e d \vec{y} \times \frac{\partial \vec{y}}{\partial t} \delta(\vec{x}-\vec{y}(t))
$$


It is readily checked that

$$
\rho=-\vec{\nabla} \cdot \vec{P}_{s}, \quad \text { and } \quad \vec{J}=\vec{\nabla} \times \vec{M}_{s}+\frac{\partial \vec{P}_{s}}{\partial t}
$$

Inserting eqs. (6) into (1), we obtain the solution for $\vec{D}$ and $\vec{H}$ of the form:

$$
\vec{D}=-\vec{\nabla} \times \vec{C}-\vec{P}_{s} \quad, \quad \vec{H}=-\vec{\nabla} C_{0}-\frac{\partial \vec{C}}{\partial t}+\vec{M}_{s}
$$

Gauss' Law and Ampere's Law (1) have thus become kinematic equations whose solution, eqs. 7 , define dual potentials $C^{\mu}=\left(C^{0}, \vec{C}\right)$.

The constitutive equations $\vec{B}=\mu \vec{H}$ and $\vec{E}=\frac{1}{\epsilon} \vec{D}=\mu \vec{D}$ determine $\vec{E}$ and $\vec{B}$ in terms of the dual potentials $C_{\mu}$ and the magnetic permeability $\mu=\frac{1}{\epsilon}$. The homogeneous Maxwell equations

$$
\vec{\nabla} \cdot \vec{B}=0, \quad \text { and } \quad \vec{\nabla} \times \vec{E}=-\frac{\partial \vec{B}}{\partial t},
$$

are then dynamical equations determining $C_{\mu}$.

As an example, consider a homogeneous medium with particles at rest. Then the equations $\vec{\nabla} \times \vec{E}=0, \vec{E}=\mu \vec{D}$, and eq. (7) give the following equation for $\vec{C}$ (denoted $\left.\vec{C}_{\text {DIRAC }} \equiv \vec{C}_{D}\right)$ :

$$
\vec{\nabla} \times\left(-\vec{\nabla} \times \vec{C}_{D}\right)=\vec{\nabla} \times \vec{P}_{s} .
$$

Comparing eqs. (9) and (4) with the equation determining the vector potential of a magnetic dipole we obtain:

$$
\vec{C}_{D}=-\int_{\vec{x}_{2}(t)}^{\vec{x}_{1}(t)} \frac{e d \vec{y}}{4 \pi} \times \frac{(\vec{x}-\vec{y})}{|\vec{x}-\vec{y}|^{3}} .
$$

Eqs. (4), (7) and (9) then yield the electric field

$$
\vec{D}=-\vec{\nabla} \times \vec{C}_{D}-\vec{P}_{s}=\vec{D}_{\mathrm{COULOMB}} \equiv \vec{D}_{\mathrm{C}}
$$

where

$$
\vec{D}_{\mathrm{C}}=\frac{e}{4 \pi}\left\{\frac{\vec{x}-\vec{x}_{1}}{\left|\vec{x}-\vec{x}_{1}\right|^{3}}-\frac{\vec{x}-\vec{x}_{2}}{\left|\vec{x}-\vec{x}_{2}\right|^{3}}\right\} .
$$

The first term $-\vec{\nabla} \times \vec{C}_{D}$ in eq. (11) produces a field analogous to the magnetic field of a line of magnetization flowing through the string. The second term $-\vec{P}_{s}$ cancels the field through the string leaving the desired pure Coulomb field. (See Fig. 2.)

For slowly moving charges, the equations $\vec{\nabla} \cdot \vec{B}=0, \vec{B}=\mu \vec{H}, \vec{\nabla} \cdot \vec{C}_{D}=0$ and eq. (7) give the following equation determining the scalar potential (denoted $C_{0 D}$ ):

$$
-\nabla^{2} C_{0 D}=-\vec{\nabla} \cdot \vec{M}_{s}
$$


Fig. 2. Diagram representing string cancellation mechanism of eq. (11)

Comparing eq. (13) with the equation determining the scalar potential of an electric dipole, and using eq. (5), we obtain:

$$
C_{0 D}=\int_{\vec{x}_{2}(t)}^{\vec{x}_{1}(t)} \frac{e d \vec{y}}{4 \pi} \times \dot{\vec{y}} \cdot \frac{(\vec{x}-\vec{y})}{|\vec{x}-\vec{y}|^{3}} .
$$

Eqs. (5), (7), (10) and (14) then yield the magnetic field

$$
\vec{H}=-\vec{\nabla} C_{0 D}-\frac{\partial \vec{C}_{D}}{\partial t}+\vec{M}_{s}=\vec{H}_{\text {Biot SAVART }} \equiv \vec{H}_{B S},
$$

where

$$
\vec{H}_{B S}=\frac{e}{4 \pi}\left[\frac{\vec{v}_{1} \times\left(\vec{x}-\vec{x}_{1}\right)}{\left|\vec{x}-\vec{x}_{1}\right|^{3}}-\frac{\vec{v}_{2} \times\left(\vec{x}-\vec{x}_{2}\right)}{\left|\vec{x}-\vec{x}_{2}\right|^{3}}\right]
$$

is the usual Biot Savart magnetic field produced by slowly moving charges.

To obtain the covariant form of the equations of the dual Maxwell theory we define the dual polarization tensor $G_{\alpha \beta}^{s}$ :

$$
G_{s k}^{s} \equiv M_{s, k}, \quad G_{i j}^{s}=-\epsilon_{i j k} P_{s, k} .
$$

The field equations (8) can then be written in the form:

$$
\partial^{\alpha} \mu\left(\partial_{\alpha} C_{\beta}-\partial_{\beta} C_{\alpha}\right)=-\partial^{\alpha} \mu G_{\alpha \beta}^{s} .
$$

These equations follow from a dual Lagrangian density $\mathcal{L}_{C}$ :

$$
\mathcal{L}_{C}=-\frac{\mu}{4}\left(\partial_{\alpha} C_{\beta}-\partial_{\beta} C_{\alpha}+G_{\alpha \beta}^{s}\right)^{2} .
$$

$\mathcal{L}_{C}$ describes the same physics as the Maxwell Lagrangian $\mathcal{L}_{A}$ :

$$
\mathcal{L}_{A}=-\frac{\epsilon}{4}\left(\partial_{\alpha} A_{\beta}-\partial_{\beta} A_{\alpha}\right)^{2}+J^{\alpha} A_{\alpha},
$$

determining the usual Maxwell equations,

$$
\partial^{\alpha} \epsilon\left(\partial_{\alpha} A_{\beta}-\partial_{\beta} A_{\alpha}\right)=J_{\beta} .
$$

If $\epsilon \rightarrow 0$ at long distances then $A_{\mu}$ is strongly coupled (anti-shielding), but since $\mu=\frac{1}{\epsilon} \rightarrow \infty, C_{\mu}$ is weakly coupled at long distances. 


\section{Non-Abelian Theory}

What is the Lagrangian density $\mathcal{L}_{\text {eff }}$ describing long-distance QCD in terms of dual potentials? Mandelstam 3 has shown that we also have the freedom to use dual potentials $C_{\mu}^{a}$ in non-Abelian gauge theory. 't Hooft ${ }^{\prime}$ showed that if the Wilson loop satisfies an area law then the dual Wilson loop (defined in terms of dual potentials) satisfies a perimeter law. This is the non-Abelian analogue of the fact that in a medium with $\epsilon<1$, dual potentials $C_{\mu}^{a}$ are screened at large distances. Although in non-Abelian gauge theory there is no direct relation between ordinary Yang Mills potentials $A_{\mu}^{a}$ and dual potentials $C_{\mu}^{a}$, we can determine the form of $\mathcal{L}_{\text {eff }}$ by imposing the following requirements:

1. $\mathcal{L}_{\text {eff }}$ must be invariant under non-Abelian gauge transformations of the $C_{\mu}^{a}$ :

$$
C_{\mu}=\Omega^{-1} C_{\mu} \Omega+\frac{i}{g} \Omega^{-1} \partial_{\mu} \Omega
$$

where $C_{\mu}=\sum_{a=1}^{8} C_{\mu}^{a} \frac{1}{2} \lambda_{a}, \Omega$ is an $S U(3)$ matrix and $g=\frac{2 \pi}{e}$ where e is the Yang Mills coupling constant, i.e. $\alpha_{s}=\frac{e^{2}}{4 \pi}$.

2. $\mathcal{L}_{\text {eff }}$ generates a mass for the $C_{\mu}$ field via a Higgs mechanism coupling $C_{\mu}$ to 3 scalar octets $B_{i}(i=1,2,3)$ carrying monopole charge.

3. We write a minimal $\mathcal{L}_{\text {eff }}$ satisfying (1) and (2).

4. We assume higher dimension operators not included in $\mathcal{L}_{\text {eff }}$ are not quantitatively important at large distances.

First consider the coupling of a quark anti-quark pair $q \bar{q}$ to $C_{\mu}$. We choose a gauge where

$$
\rho(\vec{x})=e Y\left[\delta\left(\vec{x}-\vec{x}_{1}\right)-\delta\left(\vec{x}-\vec{x}_{2}\right)\right],
$$

where $\mathrm{Y}$ is the hypercharge matrix having diagonal elements $\frac{1}{3}, \frac{1}{3},-\frac{2}{3}$ and vanishing off diagonal elements $\left(2 \operatorname{tr} Y^{2}=\frac{4}{3}\right)$. Then the covariant polarization tensor $G_{\alpha \beta}^{s}$ for the Dirac string connecting the quark anti-quark pair is given by eqs. (4), (5), and (17), multiplied by $Y$. The dual potentials $C_{\mu}$ are then also proportional to $Y$ and so $\left[C_{\mu}, C_{\nu}\right]=0$. One unit of gauge invariant $Z_{3}$ flux $e^{i \Phi}$ flows from $\bar{q}$ to $q$ along the Dirac string. To see this, evaluate

$$
e^{i \Phi} \equiv P e^{i g \oint \vec{C} \cdot d \vec{l}}
$$

where we choose the path in (24) to be a small circle surrounding the Dirac string. Near the string the effect of the coupling to the monopole fields $B_{i}$ can be neglected and $\vec{C} \sim Y \vec{C}_{D}$. Then eq. (24) becomes,

$$
e^{i \Phi}=e^{i g e Y}=e^{i 2 \pi Y}=e^{\frac{2 \pi i}{3}}
$$


Fig. 3. Quark anti-quark pair moving along trajectories $\vec{x}_{1}(t)$ and $\vec{x}_{2}(t)$, respectively.

Any continuous deformation of $\vec{C}$ in $S U(3)$ leaves $\Phi$ unchanged.

We choose a gauge for the monopole fields $B_{i}$ so that,

$$
B_{1}=\lambda_{7} B_{1}(x), \quad B_{2}=-\lambda_{5} B_{2}(x), \quad B_{3}=\lambda_{2} B_{3}(x) .
$$

As $\vec{x} \rightarrow \infty, B_{i}(\vec{x}) \rightarrow B_{0}$ where $B_{0}$ is the vacuum value of the $B_{i}$ determined by the position of the minimum of the Higgs Potential $W(B)$ :

$$
\frac{\delta W}{\delta B_{i}}=0, \quad \text { at } B_{i}=B_{0} .
$$

There is no $S U(3)$ gauge transformation which leaves invariant the vacuum values of all three $B_{i}$. Dual $S U(3)$ symmetry is then completely broken and all the dual potentials become massive.

The explicit form of the dual Lagrangian $\mathcal{L}_{\text {eff }}$ coupling a quark anti-quark pair to $C_{\mu}$ is then given by,

$$
\mathcal{L}_{\text {eff }}=2 \operatorname{tr}\left\{-\frac{1}{4}\left(\partial_{\mu} C_{\nu}-\partial_{\nu} C_{\mu}+G_{\mu \nu}^{s}\right)^{2}+\frac{1}{2}\left(\mathcal{D}_{\mu} B_{i}\right)^{2}\right\}-W
$$

where

$$
\mathcal{D}_{\mu} B_{i} \equiv \partial_{\mu} B_{i}-i g\left[C_{\mu}, B_{i}\right] .
$$

Using eqs. (26) and the fact that $C_{\mu}=Y C_{\mu}(x)$ we can eliminate the color matrices from eq. (28) to obtain

$$
\mathcal{L}_{\text {eff }}=-\frac{1}{3}\left(\partial_{\mu} C_{\nu}-\partial_{\nu} C_{\mu}+G_{\mu \nu}^{s}\right)^{2}+2\left[\left(\partial_{\mu} B_{1}\right)^{2}+\left(\partial_{\mu} B_{2}\right)^{2}+\left(\partial_{\mu} B_{3}\right)^{2}+g^{2} C^{2}\left(B_{1}^{2}+B_{2}^{2}\right)\right]-W .
$$

\section{Effective Lagrangian for Constituent Quarks}

Consider a quark (anti-quark) at $\vec{x}_{1}(t)\left(\vec{x}_{2}(t)\right)$ moving with velocities $\vec{v}_{1}(t)\left(\vec{v}_{2}(t)\right)$ (see Fig. 3). To find the effective interaction Lagrangian $L_{I}\left(\vec{x}_{1}, \vec{x}_{2} ; \vec{v}_{1}, \vec{v}_{2}\right)$ we solve the classical field equations for $C_{\mu}$ and $B_{i}$ generated by $\mathcal{L}_{\text {eff }}$, eq. (30), to first order in $\vec{v}_{1}$ and $\vec{v}_{2}$, and obtain the classical solutions:2

$$
C_{\mu}=C_{\mu}\left(\vec{x}, \vec{R}(t), \vec{v}_{1}(t), \vec{v}_{2}(t)\right) \equiv C_{\mu}^{\text {class }}, \quad B_{i}=B_{i}\left(\vec{x}, \vec{R}(t), \vec{v}_{1}(t), \vec{v}_{2}(t)\right) \equiv B_{i}^{\text {class }},
$$


Fig. 4. Static flux tube configuration

where $\vec{R} \equiv \vec{x}_{1}-\vec{x}_{2}$.

For $\vec{v}_{1}=\vec{v}_{2}=0$, the scalar potential $C_{0}^{\text {class }}=0$, and $\vec{C}^{\text {class }}$ and $B_{i}^{\text {class }}$ reduce to static flux tube solutions $\vec{C}^{\text {static }}(\vec{x}, \vec{R}), B_{i}^{\text {static }}(\vec{x}, R)$, describing a configuration where the color flux lannecting the quark anti-quark pair is confined to a tube of radius $R_{F T}$ (see Fig. 4). The monopole fields vanish at the center of the flux tube and approach their vacuum value $B_{0}$ at large distances. We insert this static solution into $\mathcal{L}_{\text {eff }}$ and integrate over all space to obtain the central potential $V_{0}(R)$ :

$$
V_{0}(R)=-\int d \vec{x} \mathcal{L}_{\text {eff }}\left(\vec{C}=\vec{C}^{\text {static }}, C_{0}=0, B_{i}=B_{i}^{\text {static }}\right) .
$$

At large $R, V_{0}(R) \rightarrow \sigma R$, where the calculated string tension $\sigma \sim\left(5 B_{0}\right)^{2}$. The experimental string tension $\sigma \sim .2 \mathrm{GeV}^{2}$ then determines $B_{0}^{2}$.

To first order in $\vec{v}_{1}(t)$ and $\vec{v}_{2}(t)$, the static distributions, $\vec{C}^{\text {static }}(\vec{x}, \vec{R})$ and $B_{i}^{\text {static }}(\vec{x}, \vec{R})$ follow the quark motion adiabatically. The time dependence of $\vec{C}^{\text {class }}$ and $B_{i}^{\text {class }}$ thus arises only from the explicit time dependence of $\vec{R}$. The resulting time varying color electric fields along with the moving Dirac string then generate a scalar potential $C_{0}^{\text {class }}$ and color magnetic fields. These produce a velocity dependent term $-V_{2}$ in the full quark anti-quark effective Lagrangian $L_{I}$ given by:

$$
L_{I}\left(\vec{x}_{1}, \vec{x}_{2} ; \vec{v}_{1}, \vec{v}_{2}\right)=\int d \vec{x} \mathcal{L}_{\text {eff }}\left(C_{\mu}=C_{\mu}^{\text {class }}, B_{i}=B_{i}^{\text {class }}\right),
$$

valid to second order in $\vec{v}_{1}$ and $\vec{v}_{2}$ since the Lagrangian is stationary about solutions to the static field equations.

Separating $L_{I}$ into its color electric and color magnetic components, we have

$$
-L_{I}\left(\vec{R}, \vec{v}_{1}, \vec{v}_{2}\right)=V_{0}(R)+V_{2}\left(\vec{R}, \vec{v}_{1}, \vec{v}_{2}\right)
$$

where the static potential $V_{0}(R)$ is determined by eq. (32). The color magnetic component $V_{2}$, obtained from eq. (33), has the form 2

$$
\begin{aligned}
V_{2}\left(\vec{R}, \vec{v}_{1}, \vec{v}_{2}\right)= & \frac{\left[\vec{R} \times\left(\vec{v}_{1}+\vec{v}_{2}\right)\right]^{2}}{4 R^{2}} V_{-}(R)+\frac{\left[\vec{R} \cdot\left(\vec{v}_{1}+\vec{v}_{2}\right)\right]^{2}}{4 R^{2}} V_{\|}(R) \\
& +\frac{\left[\vec{R} \times \frac{d \vec{R}}{d t}\right]^{2}}{4 R^{2}} V_{+}(R)+\frac{\left(\vec{R} \cdot \frac{d \vec{R}}{d t}\right)^{2}}{4 R^{2}} V_{L}(R)+V_{\text {spin }} .
\end{aligned}
$$


The spin dependent potential $V_{\text {spin }}$ includes both spin-spin and spin orbit contributions. The spin orbit contribution $\mathrm{l}^{\mathrm{a}}$ has the qualitative features of Monte Carlo data obtained from lattice gauge theory. The potentials $V_{-}(R), V_{\|}(R), V_{+}(R)$, and $V_{L}(R)$ are moments of the field distributions determined by the integrand in eq. (33). The potentials $V_{-}(R)$ and $V_{\|}(R)$ are related to $V_{0}(R)$ by Lorentz invariance: 8

$$
V_{-}(R)=-\frac{1}{2} V_{0}(R), \quad V_{\|}(R)=-\frac{1}{2} V_{0}(R)+\frac{R}{2} \frac{d V_{0}}{d R} .
$$

The function $V_{L}(R)$ determines the energy associated with longitudinal oscillations $\hat{R} \cdot \frac{d \vec{R}}{d t}$. The function $V_{+}(R)$ determines the moment of inertia $I(R)$ of the rotating flux tube according to the equation:

$$
I(R)=-\frac{1}{2} R^{2} V_{+}(R) .
$$

At large $R, V_{+}(R) \rightarrow-A R$, where $A \sim .21 \sigma$. This result for $V_{+}(R)$ along with the large $R$ result $V_{0}(R) \rightarrow \sigma R$ gives a leading Regge trajectory $\theta^{6}\left(M^{2}\right)$ which is linear for large $M^{2}$ with a slope $\alpha^{\prime} \approx \frac{1}{6.3 \sigma} \approx .75 \mathrm{GeV}^{-2}$.

We list below analytic parameterizations of the integrals over the field distributions determining $V_{0}(R), V_{+}(R)$ and $V_{L}(R)$ :

$$
\begin{gathered}
V_{0}(R)=-\frac{4}{3} \frac{\alpha_{s}}{R} e^{-.51 \sqrt{\frac{\sigma}{\alpha_{s}}} R}+\sigma R-0.65\left(\alpha_{s} \sigma\right)^{1 / 2}, \\
V_{+}(R)=-\frac{2 \alpha_{s}}{3 R} e^{-1.13 \sqrt{\frac{\sigma}{\alpha_{s}}} R}-.21 \sigma R+1.12 \sqrt{\alpha_{s} \sigma} \\
V_{L}(R)=-\frac{4 \alpha_{s}}{3 R} e^{-.68 \sqrt{\frac{\sigma}{\alpha_{s}}} R}+.09 \sqrt{\alpha_{s} \sigma} .
\end{gathered}
$$

These potentials determine generalized Wilson loops with additional electric and magnetic field insertions and hence can be compared with Monte Carlo calculations of these quantities once they are carried out.

For small $R$ the effective Lagrangian $L_{I}$ (eqs. (34)-(40)) approaches $L_{D}$, the Darwin Lagrangian (multiplied by the color factor $\frac{4}{3}$ ) describing the interaction of electrons and positrons after the elimination of the electromagnetic field. 10 Thus in the small $R$ limit $L_{I}$ gives the one gluon exchange potential to order $\left(\frac{1}{m_{q}}\right)^{2}$ just as Darwin's classical calculation gives the one photon exchange potential to the same order. $L_{I}$ can therefore be extrapolated smoothly from the large $R$ confinement region to the short distance perturbative domain. It cannot be used at shorter distances where radiative corrections giving rise to asymptotic freedom and a running coupling constant must be accounted for.

It should be emphasized that the potentials (38)-(40) are obtained from the integrated field energy associated with the color field distributions surrounding the moving 
quark anti-quark pair. As $R$ increases, the field lines are compressed and the resulting $L_{I}$ decreases less rapidly then $L_{D}$. At large $R$ the color fields evolve into a flux tube distribution and give rise to the terms in $L_{I}$ linear in $R . L_{I}$ is not calculated as a superposition of a long distance confinement contribution and a short distance perturbative term as eqs. (38)-(40) might suggest. These formulae are just analytic parameterizations of the numerical integrals described above and the $R$ dependence of these potentials reflect the evolution of the color field distributions with $R$.

Finally we note that the whole concept of an effective Lagrangian depending only upon particle positions and velocities has a meaning only when radiation can be neglected. To estimate when radiation is important, consider a quark anti-quark pair separated by a distance $R$ and moving on a circular orbit with frequency $\omega$. Then radiation occurs if $\hbar \omega>M c^{2}$, where $M$ is the mass either of the $C_{\mu}$ field or of the monopole field. (These masses are of order $500 \mathrm{MeV}$ ). Since $\omega \sim \frac{c}{R}$, this means $\hbar c / R>M c^{2}$, or $R<\hbar / M c$. Thus, for $R>\frac{\hbar}{M c} \sim R_{F T}$, radiation should be suppressed and the quark anti-quark effective Lagrangian can be used.

\section{Energy Levels of $b \bar{b}$ and $c \bar{c}$ Systems}

Starting with $L_{I}$ augmented by non-relativistic quark kinetic energy terms, we have constructed the Hamiltonian $H$ for a heavy quark anti-quark system by the canonical procedure. We determined the parameters $\alpha_{s}, \sigma, m_{c}$ and $m_{b}$ by finding a best fit to the 17 known levels of $c \bar{c}$ and $b \bar{b}$ systems. Our best fit parameters are $\alpha_{s}=.37$, $\sigma=.20 \mathrm{GeV}^{2}, m_{c}=1.34 \mathrm{GeV}, m_{b}=4.77 \mathrm{GeV}$. These values of $\alpha_{s}$ and $\sigma$ lead to a flux tube radius $R_{F T} \sim .5 \mathrm{fm}$.

\section{Summary}

(i.) We have proposed that the long distance physics of QCD is determined by a dual theory describing the interactions of dual potentials $C_{\mu}$ and monopole fields $B_{i}$ which are weakly coupled at long distances. The physical equivalence at long distances of $\mathcal{L}_{\text {eff }}\left(C_{\mu}, B_{i}\right)$ and $\mathcal{L}_{\mathrm{QCD}}$ depending upon strongly interacting $A_{\mu}$ allows us to use $\mathcal{L}_{\text {eff }}\left(C_{\mu}, B_{i}\right)$ to calculate the long distance properties of QCD (electric magnetic duality) 11

(ii.) We have used $\mathcal{L}_{\text {eff }}$ to construct an effective Lagrangian $L_{I}$ for constituent quarks, which leads to a definite quark model that can both be compared with experiment and with lattice gauge theory simulations.

\section{Acknowledgements}

I would like to acknowledge the contributions of my collaborators James S. Ball and F. Zachariasen during all stages of this work. I would also like to thank Professor H. Toki and all the organizers of this workshop for their kind hospitality.

\section{References}

1. S. Mandelstam, Phys. Rep 23C (1976) 245, G. 't Hooft, in Proceedings of the European Physical Society Conference on High Energy Physics (1976) p. 1225. 
2. M. Baker, James S. Ball and F. Zachariasen, Phys. Rev. D51 (1995) 1968.

3. S. Mandelstam, Phys. Rev. D19 (1979) 2391.

4. G. 't Hooft, Nucl. Phys. B153 (1979) 141.

5. Monte Carlo results for distributions of color flux in $S U(2)$ lattice gauge theory are given by: G. S. Bali, G Schlicter and K. Schilling, Phys. Rev. D51 (1995).

6. M. Baker, James S. Ball and F. Zachariasen, Phys. Lett. B283 (1992) 360.

7. A. Huntley and C. Michael, Nucl. Phys. B286 (1987) 211.

8. A. Barchielli, N. Brambilli and G. M. Prosperi, Nuovo Cimento 103A (1990) 59.

9. M. Baker in Proceedings of the Workshop on Quantum Infrared Physics, Paris, June 1994 (World Scientific, 1995).

10. C. G. Darwin, Phil. Mag. 39 (1920) 537.

11. It has recently been pointed out that certain supersymmetric non-Abelian gauge theories possess electric magnetic duality: N. Seiberg, Nucl. Phys. B435 (1995) 129; N. Seiberg and E. Witten, Nucl. Phys. B431 (1994) 484; N. Seiberg and E. Witten, Nucl. Phys. B426 (1994) 19. 
This figure "fig1-1.png" is available in "png" format from: http://arxiv.org/ps/hep-ph/9505346v1 
This figure "fig2-1.png" is available in "png" format from: http://arxiv.org/ps/hep-ph/9505346v1 
This figure "fig1-2.png" is available in "png" format from: http://arxiv.org/ps/hep-ph/9505346v1 
This figure "fig1-3.png" is available in "png" format from: http://arxiv.org/ps/hep-ph/9505346v1 